\title{
Adaptive Method of Formation of Parameters and Actions for Protection of Aircrafts against Man-Portable Air Defense
}

\author{
Vladimir V. Butuzov, \\ Alexander A. Dontsov* and Yuri L. Koziratsky \\ Military Training and Research Center of the Air Force \\ «Air Force Academy ft. Professor N.E. Zhukovsky and Y.A. Gagarin» \\ 54a Starykh Bol'shevikov Str., Voronezh, 394064, Russia
}

Received 14.11.2015, received in revised form 07.12.2015, accepted 16.02.2016

On the basis of the analysis of a modern level of development of means of information support of a complex of protection of aircrafts method of formation of parameters and actions for their protection against missiles of mobile surface-to-air missile systems with optical-electronic systems of homing which are based on adaptive management of temporary, spatial, power structural and frequency parameters of hindrances are formulated. Expressions for an assessment of probability of not defeat of the aircraft taking into account specifics of adaptive management of parameters of hindrances on the basis of results of control of their efficiency on trajectory and alarm signs are received. The contribution of perspective ways of adaptive management of structural and frequency parameters of hindrances allowing to increase efficiency of protection of aircrafts at group attacks by anti-aircraft guided missiles in 1,5 ... 2 times is shown.

Keywords: man-portable air defense, anti-aircraft guided missile, optical head of homing, adaptive method of creation of hindrances, efficiency control.

Citation: Butuzov V.V., Dontsov A.A., Koziratsky Y.L. Adaptive method of formation of parameters and actions for protection of aircrafts against man-portable air defense, J. Sib. Fed. Univ. Eng. technol., 2016, 9(3), 416-422. DOI: 10.17516/1999-494X2016-9-3-416-422.

(C) Siberian Federal University. All rights reserved

* Corresponding author E-mail address: addoncov1@mail.ru 


\title{
Адаптивный метод формирования параметров \\ и действий по защите воздушных судов \\ от переносных зенитных ракетных комплексов
}

\author{
В.В. Бутузов, \\ А.А. Донцов, Ю.Л. Козирацкий \\ ВУНЦ ВВС «ВВА им. проф. Н.Е. Жуковского и Ю.А. Гагарина» \\ Россия, 371600, Воронеж, ул. Старых Большевиков, 54 а
}

\begin{abstract}
На основе анализа современного уровня развития средств информационного обеспечения комплекса зашчиты воздушных судов сформулирован метод формирования параметров и действий по их защчите от ракет переносных зенитных ракетных комплексов с оптикоэлектронными системами самонаведения, которые основаны на адаптивном управлении временными, пространственными, энергетическими структурными и частотными параметрами помех. Получены выражения для оценки вероятности непоражения воздушного судна с учетом специфики адаптивного управления параметрами помех на основе результатов контроля их эффективности по траекторным и сигнальнылм признакам. Показан вклад перспективных способов адаптивного управления структурными и частотными параметрами помех, позволяющий повысить эффективность защиты воздушных судов при групповых атаках зенитныли управляемыми ракетами в 1,5 ...2 раза.
\end{abstract}

Ключевые слова: переносной зенитный ракетный комплекс, зенитная управляемая ракета, оптическая головка самонаведения, адаптивный метод создания помех, контроль эффективности.

В настоящее время решение задачи защиты воздушных судов от зенитных управляемых ракет (ЗУР) с оптическими головками самонаведения (ОГС) переносных зенитных ракетных комплексов (ПЗРК) существенно осложняется многообразием типов ЗУР с различными методами и алгоритмами обработки сигналов и выработки команд управления контуром самонаведения. Значительное распространение получили ЗУР с ОГС с растровыми модуляторами и розеточным сканированием (по западной терминологии - Con Scan и Rosette Seekers), спектральной селекцией, матричными фотоприемными устройствами (Imagers), в том числе многоспектральные (Spectral Imagers) [1]. Основное направление постоянного совершенствования ОГС - повышение эффективности поражения воздушных судов в условиях противодействия средств радиоэлектронного поражения (РЭПр), обеспечивающих оптико-электронное подавление или функциональное поражение ОГС.

Существенное разнообразие средств поражения привело к увеличению количества и расширению номенклатуры датчиков угроз и средств противодействия, одновременно размещаемых на борту защищаемого воздушного судна. При этом жесткие массогабаритные и энергетические ограничения привели к необходимости комплексирования средств защиты и их интеграции в состав бортового радиоэлектронного оборудования. Создана оптикоэлектронная подсистема комплекса радиоэлектронного подавления, включающая информационную подсистему (аппаратуру обнаружения лазерного излучения, ультрафиолетовые и инфракрасные датчики обнаружения пуска и сопровождения атакующих ракет) и исполнительную подсистему (устройства выброса ложных тепловых целей, когерентные и некоге- 
рентные станции активных помех) [2]. Особенность оптико-электронной подсистемы - открытая архитектура и высокие возможности спецвычислителя устройства управления, позволяющего реализовать различные алгоритмы работы комплекса с учетом оценки и анализа степени реальных угроз. При этом весьма актуальна задача формирования эффективных помех в условиях отсутствия информации о типе и параметрах ОГС ЗУР, которая может быть решена в рамках адаптационного подхода и базироваться (с учетом специфики применения ЗУР) на основе прямого способа восстановления неизвестных характеристик объектов подавления [3-6].

Цель работы - разработка адаптивного метода формирования параметров и действий по радиоэлектронному поражению оптических головок самонаведения ЗУР ПЗРК для повышения эффективности защиты воздушных судов.

Предпосылками создания адаптивного метода формирования параметров и действий по РЭПр являются созданные к настоящему времени высокотехнологичные средства информационного обеспечения и средства создания помех с изменяемыми параметрами, управляемые в реальном масштабе времени. В качестве параметров адаптации могут использоваться временные, пространственные, структурные, энергетические и частотные параметры, вклад которых в формирование адаптивного метода РЭПр представлен на рис. 1. Как видим, для формирования адаптивного метода РЭПр используется множество информационных параметров, получаемых с помощью информационных каналов оптико-электронной подсистемы комплекса защиты воздушного судна. С помощью пассивных каналов могут определяться время пуска ЗУР и ее текущие угловые координаты, с помощью активных - дальность до ЗУР, угловые координаты ОГС, структура и параметры отраженного от ОГС сигнала лазерного локатора. Возможность получения информации от пассивных и активных каналов определяют 1-й и 2-й уровни адаптации при формировании метода РЭПр ОГС ЗУР.

На первом уровне адаптации достаточно получение информации только от пассивных каналов. В этом случае происходит программное управление средствами создания помех, реализующих способы создания помех с заданными структурными, временными и пространственными параметрами. Управление энергетическими параметрами в данном случае может осуществляться по результатам контроля эффективности помех на основе текущих наблюдений изменения угловых координат атакующих ЗУР. Программное управление средствами создания помех в данном случае должно базироваться на прогнозных моделях оценки эффективности помех с заданными пространственными, частотными и энергетическими характеристиками в условиях априорной неопределенности о типе и параметрах ОГС. Фактически на первом уровне адаптации формируется набор прицельных по времени и направлению активных и пассивных помех с заранее заданными структурой и частотными характеристиками, позволяющими обеспечить эффективное РЭПр ЗУР с ОГС определенного типа.

При этом адаптация по энергетическому параметру в данном случае подразумевает повышение мощности активного помехового канала при приближении ЗУР к воздушному судну на расстояние, позволяющее осуществить функциональное поражение ее ОГС.

Приоритет угрозы, позволяющий определить первоочередные объекты РЭПр при групповой атаке ЗУР, на первом уровне адаптации будет определяться временем пуска ЗУР и коэффициентом угроз, определяемым выражением 


$$
K_{\mathrm{y \Gamma} 1}=\int_{T_{\mathrm{\Pi}}}^{t} v_{\mathrm{y \Gamma}}(t) d t / \pi
$$

где $v_{\text {уг }}$-угловая скорость сопровождения ЗУР, $T_{\Pi}-$ время пуска ЗУР.

В таком случае наибольшую угрозу при одновременной атаке будет представлять ЗУР с наименьшим значением $K_{\mathrm{yг}}^{1}$.

Второй, более высокий, уровень адаптации предусматривает получение информации от пассивных и активных каналов. При этом активные каналы используются для определения дальности до атакующей ЗУР, что позволяет реализовать эшелонированное создание помех и адаптивное управление их временными и энергетическими параметрами, а также для уточнения угловых координат ОГС. Дополнительно определяются структура и частотные параметры отраженного от ОГС зондирующего сигнала, позволяющие реализовать адаптивные способы создания помех ЗУР с ОГС различных типов и контролировать эффективность помех по результатам текущих не только траекторных, но и сигнальных измерений.

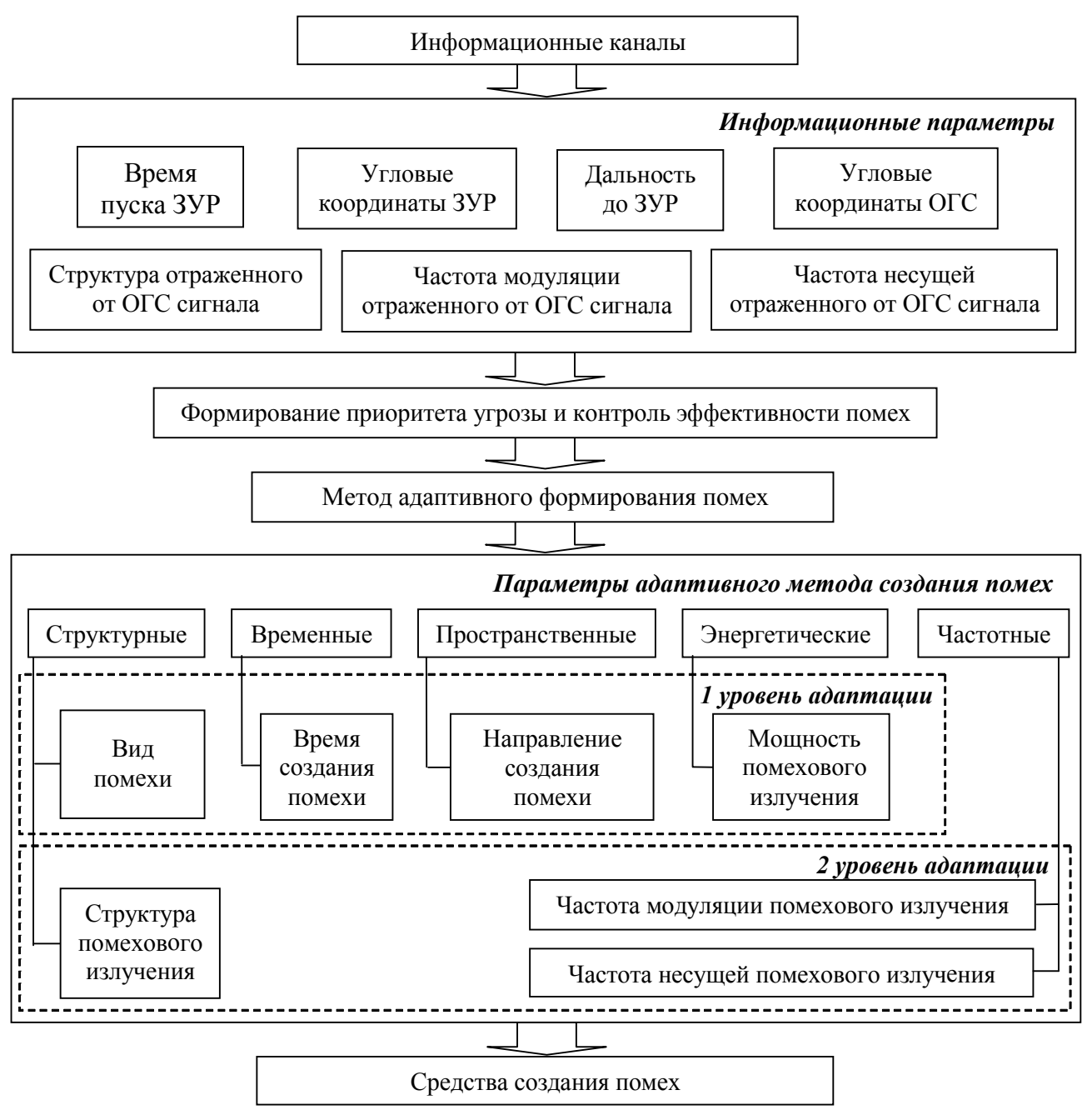

Рис. 1. Структура адаптивного метода радиоэлектронного поражения

$$
-419-
$$


Приоритет угрозы на втором уровне адаптации целесообразно определять минимальным значением коэффициента, равного отношению текущей дальности до атакующей ЗУР $D(t)$ к дальности ее пуска $\mathrm{D}_{\Pi}=D\left(T_{\mathrm{H}}\right)$ :

$$
K_{\text {уг } 2}=\frac{D(t)}{D_{\text {п }}}
$$

Проведем гипотетическую оценку влияния используемых информационных параметров на 1-м и 2-м уровнях адаптации на эффективность решения задачи защиты воздушного судна от ЗУР с ОГС различных типов. Вероятность непоражения воздушного судна в результате $N_{\text {a }}$ атак ЗУР определяется выражением

$$
P_{\mathrm{H \Pi}}=\prod_{i=1}^{N_{\mathrm{a}}}\left(1-P_{\Pi}^{i}\right),
$$

где $P_{\Pi}^{i}-$ вероятность поражения воздушного судна при $i$-й атаке.

Вероятность $P_{\Pi}^{i}$ обусловлена вероятностью применения ЗУР с $j$-м типом ОГС $P_{j}$ и вероятностью непоражения воздушного судна ЗУР с $j$-тым типом ОГС в результате $i$-й атаки $P_{\mathrm{H}}^{i j}$ (в том числе и в условиях помех):

$$
P_{\Pi}^{i}=\sum_{j=1}^{N_{\mathrm{T}}} P_{j}\left(1-P_{\mathrm{H} \Pi}^{i j}\right),
$$

где $N_{\text {T }}$ - число типов ОГС ЗУР.

При реализации адаптивных методов РЭПр $P_{\mathrm{H \Pi}}^{i j}$ равна

$$
P_{\mathrm{H} \Pi}^{i j}=P_{\mathrm{об} \mathrm{H}}^{i}\left(P_{\mathrm{Haв}}^{i} P_{\mathrm{H} \Pi 1}^{j}+\left(1-P_{\mathrm{Ha \textrm {B }}}^{i} P_{\mathrm{H} \Pi 1}^{j}\right) P_{\mathrm{H} 2}^{j}\right),
$$

где $P_{\text {обн }}^{i}$ - вероятность обнаружения пуска ЗУР при $i$-й атаке; $P_{\text {нав }}^{i}-$ вероятность наведения активного канала противодействия при $i$-й атаке; $P_{\text {нп1 }}^{j}-$ вероятность непоражения воздушного судна ЗУР с ОГС $j$-го типа при применении когерентных или некогерентных источников помех; $P_{\text {нп2 }}^{j}$ - вероятность непоражения воздушного судна ЗУР с ОГС $j$-го типа при применении расходуемых средств создания помех.

С учетом влияния расходуемых средств создания помех на функционирование пассивных информационных каналов $[7,8]$ при $i$-й атаке для 1-го уровня адаптации, когда используется программное применение всего набора расходуемых средств создания помех, вероятности обнаружения и наведения канала противодействия:

$$
P_{\text {обн(нав) }}^{i}=(1-\alpha(i-1) / \pi) P_{\text {обн(нав })}^{0},
$$

где $P_{\text {обн(нав) }}^{0}-$ вероятность обнаружения пуска ЗУР до применения расходуемых средств создания помех; $a$ - сектор обзора пассивного информационного канала, «закрытый» излучением расходуемых помеховых источников.

С помощью выражений (3)-(6) проведена оценка вклада в приращение вероятности непоражения воздушного судна $W$ предложенного адаптивного метода создания помех второго, более высокого уровня, за базовый принят первый уровень адаптации. Расчеты проведены при следующих значениях исходных данных: $N_{\mathrm{T}}=4 ; P_{\text {обн }}^{0}=0,9 ; P_{\text {нав }}^{0}=0,95 ; \alpha=\pi / 6 ; P_{j}=1 / N_{\mathrm{T}}$;

$$
-420-
$$




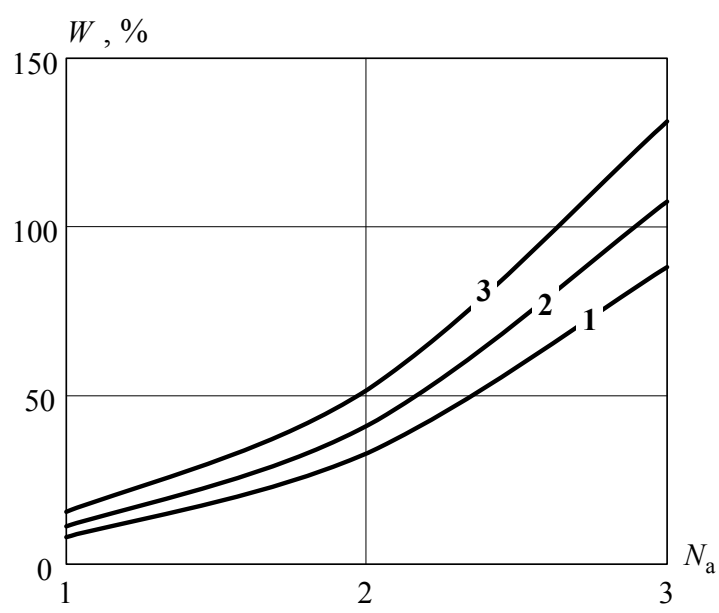

Рис. 2. Зависимости приращения вероятности непоражения воздушного судна от числа атак ЗУР

$P_{\text {нп1 }}^{j}=0,8 ; P_{\text {нп2 }}^{j}=0,7$. На рис. 2 представлены зависимости приращения вероятности непоражения защищаемого воздушного судна $W$ от числа атак ЗУР, рассчитанные в предположении возможного увеличения на 2-м уровне адаптации вероятностей $P_{\text {нп1 }}^{j}$ и $P_{\mathrm{Hп} 2}^{j}$ на 5,10 и $15 \%$ (кривые 1,2 и 3 соответственно).

Анализ графиков рис. 2 показывает, что увеличение числа параметров адаптации позволяет повысить вероятность непоражения воздушного судна в дуэльной ситуации на $10 \ldots 15 \%$ при парных пусках ЗУР - на 30...50 \% и при атаке трех ЗУР - на 90..130 \%.

Таким образом, достигнутый к настоящему времени уровень развития средств информационного обеспечения комплексов защиты воздушных судов позволяет обеспечить формирование адаптивного метода радиоэлектронного поражения оптических головок самонаведения современных ЗУР ПЗРК с принципиально различными методами и алгоритмами обработки сигналов и выработки команд управления контуром самонаведения.

Адаптивное управление временными, пространственными, энергетическими, структурными и частотными параметрами помех позволит существенно, в 1,5...2 раза, повысить эффективность защиты воздушных судов при одновременных атаках нескольких ЗУР с различными типами оптических головок самонаведения.

\section{Список литературы}

[1] А. Канов, П. Алексеев // Зарубежное военное обозрение. 2012. № 5. С. 46-50. [Kanov A., Alekseev P. Zarubezhnoe voennoe obozrenie, 2012, 5, 46-50. (in Russian)]

[2] Бутузов В.В. // Материалы VII отраслевой научно-практической конференции «Радиоэлектронные технологии: состояние и перспективы развития». Воронеж: Печатный дом, 2009. C. 140-149. [Butuzov V.V. Materials of VII branch scientific and practical conference "Radioelectronic technologies: state and prospects of development", Voronej, Pechatnyi dom, 2009, 140-149. (in Russian)]

[3] Козирацикии Ю.Л., Юхно П.М. // Радиотехника. 2000. № 8. C. 4-11. [Koziratskii Yu.L., Yuhno P.M., Radioengineering, 2000, 8, 4-11. (in Russian)] 
[4] Модели информационного конфликта средств поиска и обнаружения / под ред. Ю. Козирацкого. М.: Радиотехника, 2013. 232 с. [Models of information conflict of means of search and detection. Edited by Yu.L. Koziratskii, Moscow, Radioengineering, 2013, 232 p. (in Russian)]

[5] Модели пространственного и частотного поиска / под ред. Ю. Козирацкого. М.: Радиотехника, 2014. 344 c. [Models of spatial and frequency search. Edited by Yu.L. Koziratskii, Moscow, Radioengineering, 2014, 344 p. (in Russian)]

[6] Обнаружение и координатометрия оптико-электронных средств, оценка параметров их сигналов / под ред. Ю. Козирацкого. М.: Радиотехника, 2015. 456 с. [Detection and koordinatometriya of optical-electronic means, assessment of parameters of their signals. Edited by Yu.L. Koziratskii, Moscow, Radioengineering, 2015, 456 p. (in Russian)]

[7] Козираикий Ю.Л., Прохоров Д.В., Плеве В.В. // Радиотехника. 2011. № 8. С. 26-30. [Koziratskii Yu.L., Prohorov D.V., Pleve V.V., Radioengineering, 2011, 8, 26-30. (in Russian)]

[8] Донцов А.А., Козираикий Ю.Л. // Автометрия. 2013. Т. 49. № 2. С. 42-48. [Dontsov A.А., Koziratskii Yu.L., Avtometriia, 2013, Vol. 49, 2, 42-48. (in Russian)] 